\title{
The Pythagoras number of real sum of squares polynomials and sum of square magnitudes of polynomials
}

\author{
Thanh Hieu Le · Laurent Sorber · Marc Van Barel
}

Received: 14 March 2012 / Accepted: 20 August 2012

(C) Springer-Verlag 2012

\begin{abstract}
In this paper, we conjecture a formula for the value of the Pythagoras number for real multivariate sum of squares polynomials as a function of the (total or coordinate) degree and the number of variables. The conjecture is based on the comparison between the number of parameters and the number of conditions for a corresponding low-rank representation. This is then numerically verified for a number of examples. Additionally, we discuss the Pythagoras number of (complex) multivariate Laurent polynomials that are sum of square magnitudes of polynomials on the $n$-torus. For both types of polynomials, we also propose an algorithm to numerically compute the Pythagoras number and give some numerical illustrations.
\end{abstract}

Keywords Pythagoras number - Sum of squares polynomials - Sum of square magnitudes of polynomials $\cdot$ Low-rank representation

Mathematics Subject Classification $11 \mathrm{E} 25 \cdot 12 \mathrm{Y} 05 \cdot 65 \mathrm{H} 10$

\footnotetext{
The research was partially supported by the Research Council KU Leuven, project OT/10/038 (Multi-parameter model order reduction and its applications), PF/10/002 Optimization in Engineering Centre (OPTEC), and by the Interuniversity Attraction Poles Programme, initiated by the Belgian State, Science Policy Office, Belgian Network DYSCO (Dynamical Systems, Control, and Optimization). Laurent Sorber is supported by a Flanders Institute of Science and Technology (IWT) doctoral scholarship. The scientific responsibility rests with its author(s).
}

T. H. Le · L. Sorber · M. Van Barel ( $\varangle)$ Department of Computer Science, KU Leuven, Celestijnenlaan 200A, 3001 Heverlee, Belgium e-mail: marc.vanbarel@cs.kuleuven.be s

T. H. Le

e-mail: thanhhieu.le@cs.kuleuven.be

L. Sorber

e-mail: laurent.sorber@cs.kuleuven.be 


\section{Introduction}

Let $f$ be one of the two following polynomials:

- sum of squares polynomial in $n$ real variables $x_{1}, x_{2}, \ldots, x_{n}$

$$
f=\sum_{i=1}^{m} g_{i}^{2}
$$

with real coefficients and of total or coordinate degree $2 d ; g_{i}$ is a real-coefficient polynomial of total or coordinate degree at most $d$, for all $i=1, \ldots, m$, corresponding to the degree of $f$.

- sum of square magnitudes of polynomials in $n$ complex variables $z_{1}, z_{2}, \ldots, z_{n}$ on the $n$-torus $\mathbb{T}^{n}=\left\{\left(z_{1}, \ldots, z_{n}\right):\left|z_{i}\right|=1, \forall i=1, \ldots, n\right\}$

$$
f=\sum_{i=1}^{m}\left|g_{i}\right|^{2}
$$

with complex coefficients and of total or coordinate degree $d$; the complexcoefficient polynomials $g_{i}$ are of total or coordinate degree at most $d$.

We will write sos-polynomial and sosm-polynomial for polynomials of the form (1) and (2), respectively. Note that $m$ can be arbitrarily large. For different values of $n$ and $d$, we look for the representation of $f$ by a minimum number $\pi(f)$ of $\operatorname{sos}(\mathrm{m})$-terms, i.e., in case of a sos-polynomial

$$
\begin{aligned}
f & =\sum_{i=1}^{m} g_{i}^{2} \\
& =\sum_{i=1}^{\pi(f)} h_{i}^{2}
\end{aligned}
$$

and in a similar way for sosm-polynomials (2). It turns out that the number $\pi(f)$, called Pythagoras number, does not grow much for increasing values of the number of variables and the degree of the polynomial $f$.

In several applications that can be written in terms of sos(m)-polynomials, it is desirable to know the precise value of the Pythagoras number. Such polynomials can be represented as a sum of squares (or sum of square magnitudes of polynomials) with a number of terms not greater than the number of sos(m)-terms in the initial representation of the polynomial. It follows that, the sos(m)-polynomials can be represented by a "low-rank representation" (see Sects. 2 and 6.1), leading to a reduction in computational complexity.

In this paper, we will conjecture a formula for the value of the Pythagoras number $\pi_{T}(n, 2 d), \pi_{C}(n, 2 d)$ of the sos-polynomials for the two cases of total and coordinate degree, respectively, in Sect. 3. The conjectured value is determined by comparing the number of parameters in the low-rank representation of sos-polynomials and the 
number of conditions on these parameters. In Sect. 4 we develop an algorithm to numerically compute the Pythagoras number for a given sos-polynomial. In Sect. 5 , we use this algorithm to compute the Pythagoras number $\left(\pi_{T}(f)\right.$ for total degree and $\pi_{C}(f)$ for coordinate degree) for different samples of $f$, with $f$ a random sospolynomial in $\mathbb{R}[x]_{2 d}$. This gives a lower bound for the Pythagoras number of the whole set $\mathbb{R}[x]_{2 d}$. It turns out that these Pythagoras numbers are much smaller than the upper bounds for the corresponding sets. In fact, one can see that they are determined as the ceil of the lower bound. For example, $\pi_{T}(n, 2 d)=\left\lceil\lambda_{T}(n+1,2 d)\right\rceil$ (see Sect. 3).

These facts are shown in Tables 1 and 2 of Sect. 5. A discussion on the Pythagoras number of (complex) Laurent polynomials positive on the $n$-torus is given in Sect. 6 . The Laurent polynomials in that section are considered as sum of square magnitudes of complex-coefficient polynomials. Sect. 6.1 summarizes the link between sum of square magnitudes representation of Laurent polynomials and their matrix forms. Sect. 6.2 presents a conjecture on the Pythagoras number of sosm-polynomials and introduces how to apply the generalized Levenberg-Marquardt algorithm [18] to solve the corresponding system of quadratic polynomial equations. In the last subsection we use this algorithm to compute the Pythagoras number for different samples of sum of square magnitudes Laurent polynomials. Section 7 gives the conclusion.

\section{Sum of squares polynomials via low-rank representation}

In this section, we summarize a well-known relation between the coefficients of a polynomial which can be represented as a sum of squares and the coefficients of the terms. Using the Gram-matrix representation [5, §2], the "low-rank" representation of such a sos-polynomial is obtained. Let $\Omega(n, d)$ be a finite subset, containing zero, of a halfspace in $\mathbb{Z}^{n}$. Recall that a halfspace in $\mathbb{Z}^{n}[10]$ is a subset $S$ such that $S \cap(-S)=$ $\{0\}, S \cup(-S)=\mathbb{Z}^{n}$ and $S+S \subseteq S$. For example, a standard halfspace of $\mathbb{Z}$ is $E_{1}=\mathbb{N}$ and the one of $\mathbb{Z}^{n}$ is $E_{n}=\left(\{0\} \times E_{n-1}\right) \cup\left(\mathbb{N} \backslash\{0\} \times \mathbb{Z}^{n}\right), n \geq 2$. Then the set of all exponents of the monomials of the polynomial $g_{i}$ in $(1)$ is $\Omega(n, d)$ and the set of all exponents of $f$ is defined by

$$
\Gamma^{\Re}(n, d)=\Omega(n, d)+\Omega(n, d)=\{\alpha+\beta: \alpha, \beta \in \Omega(n, d)\} .
$$

Let $\omega, \bar{\omega}$ be the cardinality of $\Omega(n, d)$ and $\Gamma^{\Re}(n, d)$, respectively.

For the case of total degree, we have

$$
\begin{aligned}
& \Omega_{T}(n, d)=\left\{\alpha=\left(\alpha_{1}, \ldots, \alpha_{n}\right) \in \mathbb{N}^{n}: 0 \leq|\alpha|=\sum_{i=1}^{n} \alpha_{i} \leq d\right\}, \\
& \Gamma_{T}^{\Re}(n, d)=\left\{\gamma \in \mathbb{N}^{n}:|\gamma| \leq 2 d\right\} .
\end{aligned}
$$

For the case of coordinate degree, we have

$$
\begin{aligned}
& \Omega_{C}(n, d)=\left\{\alpha \in \mathbb{N}^{n}: 0 \leq \alpha_{i} \leq d, \quad \forall i=1, \ldots, n\right\}, \\
& \Gamma_{C}^{\Re}(n, d)=\left\{\gamma \in \mathbb{N}^{n}: \quad 0 \leq \gamma_{i} \leq 2 d, \quad \forall i=1, \ldots, n\right\} .
\end{aligned}
$$


Note that the number of elements of $\Omega_{T}(n, d), \Gamma_{T}^{\Re}(n, d), \Omega_{C}(n, d)$, and $\Gamma_{C}^{\Re}(n, d)$ are $\hat{e}=e(n+1,2 d), \hat{a}=a(n+1,2 d),(d+1)^{n}$ and $(2 d+1)^{n}$, respectively, where

$$
e(n, 2 d)=\frac{(n+d-1) !}{(n-1) ! d !}, \quad a(n, 2 d)=\frac{(n+2 d-1) !}{(n-1) !(2 d) !} .
$$

We will write $\Omega(n, d)$ and $\Gamma^{\mathfrak{R}}(n, d)$ for both cases of total and coordinate degree, unless stated otherwise.

From now on, we take $n, d$ as two natural numbers which are greater than 1 . Let $\mathbb{R}\left[x_{1}, \ldots, x_{n}\right]$ denote the commutative ring of all polynomials with real coefficients in $n$ variables $x_{1}, \ldots, x_{n}$. In case the natural number $n$ is fixed, we simplify the notation by writing $\mathbb{R}[x]=\mathbb{R}\left[x_{1}, \ldots, x_{n}\right]$. Let

$$
\mathbb{R}[x]_{2 d}=\{f \in \mathbb{R}[x]: \operatorname{deg}(f) \leq 2 d\},
$$

where $\operatorname{deg}(f)$ denotes the (total or coordinate) degree of the polynomial $f$ and $d \in \mathbb{N}$.

Definition 1 (Pythagoras number of real polynomials) The Pythagoras number of a sum of squares polynomial $f \in \mathbb{R}[x]_{2 d}$, denoted as $\pi(f)$, is defined to be the smallest positive integer such that $f$ can be represented as a sum of $\pi(f)$ squares of elements in $\mathbb{R}[x]$. This is also called the length of $f[5,14]$. The Pythagoras number of a subset $\emptyset \neq S \subseteq \mathbb{R}[x][5,14]$ is defined to be the supremum of the Pythagoras numbers of all sum of squares elements in $S$.

Note that the number $\pi(f)$ is always less than or equal to the Pythagoras number of the set $\mathbb{R}[x]_{2 d}$. In the case $S=\mathbb{R}[x]_{2 d}$, the definition of the Pythagoras number $\pi(S)$ above coincides the one in [2, Definition 6.4.1].

For the case of the total degree, some results on the Pythagoras number of the two sets

$$
\mathbb{H}[x]_{2 d}=\left\{f \in \mathbb{R}[x]_{2 d}: f \text { is homogeneous of total degee } 2 d\right\}
$$

and $\mathbb{R}[x]_{2 d}$ can be summarized as follows. Let $p_{T}(n, 2 d)$ and $\pi_{T}(n, 2 d)$ denote the Pythagoras number of $\mathbb{H}[x]_{2 d}$ and $\mathbb{R}[x]_{2 d}$, respectively. Concerning the first one, Robinson [16] proved that

$$
p_{T}(n, 2 d) \leq a(n, 2 d) .
$$

Choi et al. [5] derived the sharper upper bound $\Lambda_{T}(n, 2 d)$ and also the lower bound $\lambda_{T}(n, 2 d)$ satisfying the inequality

$$
\frac{a(n, 2 d)}{e(n, 2 d)} \leq \lambda_{T}(n, 2 d) \leq p_{T}(n, 2 d) \leq \Lambda_{T}(n, 2 d) \leq e(n, 2 d) \leq a(n, 2 d),
$$


where

$$
\begin{aligned}
& \lambda_{T}(n, 2 d)=\frac{2 e(n, 2 d)+1-\sqrt{[2 e(n, 2 d)+1]^{2}-8 a(n, 2 d)}}{2}, \\
& \Lambda_{T}(n, 2 d)=\frac{\sqrt{1+8 a(n, 2 d)}-1}{2} .
\end{aligned}
$$

Moreover, the upper bound $\Lambda_{T}(n, 2 d)$ can be obtained from [1, Theorem 1.3]. More specifically, if $f$ is homogeneous of total degree $2 d$ then $f$ has $a(n, 2 d)$ coefficients. Then, using the Gram-matrix representation of this polynomial (see Sect. 2), the identification of the coefficients of $f$ in the initial representation and matrix one gives us the conditions in the hypothesis of [1, Theorem 1.3]. The conclusion of this theorem gives us the upper bound $\Lambda_{T}(n, 2 d)$ of the Pythagoras number.

This bound can also be derived from [12, Theorem 2.1 (1)].

For the case $\mathbb{R}[x]_{2 d}$, the following upper bound is obtained in [14, Chapter 8]

$$
\pi_{T}(n, 2 d) \leq e(n+1,2 d) .
$$

As far as we know, the problem of determining the exact value of Pythagoras numbers $p_{T}(n, 2 d)$ and $\pi_{T}(n, 2 d)$ of the two sets above are still open except for some special cases: $p_{T}(3,4)=3[8,15]$ and $\pi_{T}(1,2 d)=2$ for all $d \geq 1[4,9,14]$. The following proposition gives us a connection between the Pythagoras number of $\mathbb{H}[x]_{2 d}$ and the one of $\mathbb{R}[x]_{2 d}$.

Proposition 1 Let $f(x)=\sum_{\gamma \in \Gamma^{\Re}(n, d)} f_{\gamma} x^{\gamma} \in \mathbb{R}[x]_{2 d}$ be a polynomial of total degree $2 d$ in $n$ variables. Then $f(x)$ is a sos-polynomial if and only if its homogenization

$$
\bar{f}(x, y)=\sum_{\gamma \in \Gamma^{\Re}(n, d)} f_{\gamma} x^{\gamma} y^{2 d-|\gamma|}, \quad y \in \mathbb{R}
$$

is a homogeneous sos-polynomial.

A corollary of Proposition 1 is that

$$
\pi_{T}(n, 2 d)=p_{T}(n+1,2 d), \quad \forall n \geq 1, \quad \forall d \geq 1
$$

It is clear that $p_{T}(2,2 d)=\pi_{T}(1,2 d)=2, \forall d \geq 1$. In this case, $\lambda_{T}(2,2 d)=$ $2, \forall d \geq 1$, (see also [8,15]). One can see that $\pi_{T}(n, 2) \geq n+1$ since [2, Corollary 6.4.8] the polynomial $1+x_{1}^{2}+\cdots+x_{n}^{2}$ is not a sum of $n$ squares in $\mathbb{R}[x]$, and moreover, $e(n+1,2)=n+1$, we have $\pi_{T}(n, 2)=p_{T}(n+1,2)=n+1, \forall n \geq 1$ (see also $[8,15])$.

For the coordinate degree, since each $\gamma \in \Gamma_{C}(n, d),|\gamma| \leq 2 n d$ and this is valid for the elements of $\Omega_{C}(n, d)$. That is, $|\alpha| \leq n d$ for any $\alpha \in \Omega_{C}(n, d)$. The following proposition gives us a relation between the Pythagoras number for the total degree and the one for the coordinate degree. 
Proposition 2 (i) Any sos-polynomial $f \in \mathbb{R}[x]$ in $n$ variables and of coordinate degree $2 d$ is always a sos-polynomial of total degree $2 n d$;

(ii) $\pi_{C}(f)=\pi_{T}(f)$;

(iii) $\pi_{T}(n, 2 d) \leq \pi_{C}(n, 4 d) \leq \pi_{T}(n, 4 n d)$.

Let $f(x)=\sum_{\gamma \in \Gamma^{\Re}(n, d)} f_{\gamma} x^{\gamma} \in \mathbb{R}[x]_{2 d}$ be written in terms of the monomials $x^{\gamma}=x_{1}^{\gamma_{1}} \cdots x_{n}^{\gamma_{n}}$. We see that a polynomial $f(x)=\sum f_{\gamma} x^{\gamma} \in \mathbb{R}[x]_{2 d}$ which is a sum of $r$ squares $h_{j}(x)^{2}$ can be represented in matrix form as

$$
f(x)=\sum f_{\gamma} x^{\gamma}=\mathbf{x}_{2 d}^{T} \mathbf{f}=\sum_{j=1}^{r} h_{j}(x)^{2}=\mathbf{x}_{d}^{T}\left(H H^{T}\right) \mathbf{x}_{d},
$$

where $\mathbf{x}_{d}$ denotes the column vector of monomials $x^{\gamma}$ of degree not greater than $d$ arranged in a given order, $\mathbf{x}^{T}$ denotes the transposed vector of $\mathbf{x}, \mathbf{f}$ is the column vector of the corresponding coefficients $f_{\gamma}$ and $H$ is the matrix whose $j$ th column is the vector of coefficients $\mathbf{h}_{j}$ of the polynomial $h_{j}(x)$ corresponding to the ordering in the vector $\mathbf{x}_{d}$. Hence, we obtain a low-rank representation for the sos-polynomial $f(x)$ with $\operatorname{rank}(H) \leq r$. So, from (8) we get a system of non-linear (quadratic polynomial) equations in variables $h_{i j}$ 's

$$
\sum_{\alpha+\beta=\gamma}\left(\sum_{k=1}^{r} h_{k \alpha} h_{k \beta}\right)=f_{\gamma}, \forall \gamma \in \Gamma^{\Re}(n, d),
$$

where $\alpha, \beta \in \Omega(n, d)$.

If the entries of the associated matrix $X=H H^{T}$ of the sos-polynomial $f$ are viewed as the parameters, i.e., (9) is a system of linear equations where the unknowns are the entries of $X$, then one can see from [5] that $X$ can be found as a linear combination of $(\hat{e} \times \hat{e})$-symmetric matrices: $X=X_{0}+t_{1} X_{1}+\cdots+t_{\delta} X_{\delta}$, where $\delta=\frac{\hat{e}(\hat{e}+1)}{2}-\hat{a}$ and $t_{1}, \ldots, t_{\delta}$ are parameters. All the eigenvalues of $X$ are nonnegative because of the positive semidefiniteness of $X$. Moreover, the eigenvalues as well as the coefficients of the characteristic polynomial depend on the parameters $t_{1}, \ldots, t_{\delta}$. Using this combination and applying Descartes' rule of signs of the characteristic polynomial, Powers and Wörmann [13] proposed an algorithm for checking whether a polynomial is sos. The minimal rank of $X$ hence is determined for some $\delta$-tuple $\left(t_{1}, \ldots, t_{\delta}\right)$. This is however difficult to do as soon as the degree of the polynomial and the number of variables becomes large.

We would now like to check whether a sos-polynomial $f(x)=\sum f_{\gamma} x^{\gamma} \in \mathbb{R}[x]_{2 d}$ can be represented as a sum of squares in which the number of terms in the sum is smaller than the one in the initial representation. The unknowns in our implementation are the entries of $H$ (not the ones of $H H^{T}$ ). Suppose $f$ is sos as in (1). We note that $\pi(f)$ is the minimal value of the rank of all associated matrices $\left(H H^{T}\right)$ of $f$ as in (8). This means $\pi(f) \leq \omega$. It is sufficient to assume that $f(x)$ is given as a sum of $\omega$ squares in $\mathbb{R}[x]_{2 d}$, i.e., $m=\omega$. In our algorithm in Sect. 4 , we check step-bystep whether $f(x)$ is a sum of $r=1,2, \ldots, \omega$ squares. More precisely, we solve the system of nonlinear equations (9) in unknowns $h_{i j}$ 's for each $r=1,2, \ldots, \omega$. The 
first natural number $r \leq \omega$ such that the above system has a solution is the one we want to find. This is the Pythagoras number of the given sos-polynomial and it gives us a "low-rank" representation of the polynomial.

\section{Conjecture}

In the previous section, we have derived the low-rank representation (8) for a sospolynomial based on the matrix $H$ having $\omega$ rows and $r$ columns, $r \leq \omega$. Hence, we need $\omega r$ parameters to represent such a sos-polynomial. However, not all choices of these parameters lead to a different sos-polynomial. Assuming that $H$ is full rank, there is a square $r \times r$ submatrix $A$ of $H$ that is nonsingular. Taking the $L Q$ factorization of $A$, we can transform $H$ into the matrix $\tilde{H}$ as

$$
\tilde{H}=H Q^{T}
$$

having the part corresponding to $A$ equal to $L$. Hence, instead of having $\omega r$ parameters, the number of free parameters $\mu(\omega, r)$ to represent a rank $r$ positive semidefinite matrix of size $\omega \times \omega$ is equal to

$$
\mu(\omega, r)=\omega r-\frac{r(r-1)}{2} .
$$

The number of conditions in (9) that have to be satisfied for these parameters is equal to the number of elements of $\Gamma^{\Re}(n, 2 d)$, i.e., $\bar{\omega}$. Hence, as soon as the number of conditions $\bar{\omega}$ is not larger than the number of free parameters $\mu(\omega, r)$, we expect to have a solution of the system of nonlinear equations (9).

Conjecture 1 The Pythagoras number $\pi(f)$ is the smallest number $r$ such that

$$
\mu(\omega, r)=\omega r-\frac{r(r-1)}{2} \geq \bar{\omega}
$$

Equivalently,

$$
r=\left\lceil\frac{2 \omega+1-\sqrt{(2 \omega+1)^{2}-8 \bar{\omega}}}{2}\right\rceil
$$

It is not difficult to see that condition (10) is equivalent to

$$
0<\frac{2 \omega+1-\sqrt{(2 \omega+1)^{2}-8 \bar{\omega}}}{2} \leq r \leq \frac{2 \omega+1+\sqrt{(2 \omega+1)^{2}-8 \bar{\omega}}}{2}
$$

So, the Pythagoras number of polynomial $f$ in total degree and coordinate degree according to our conjecture are equal to 

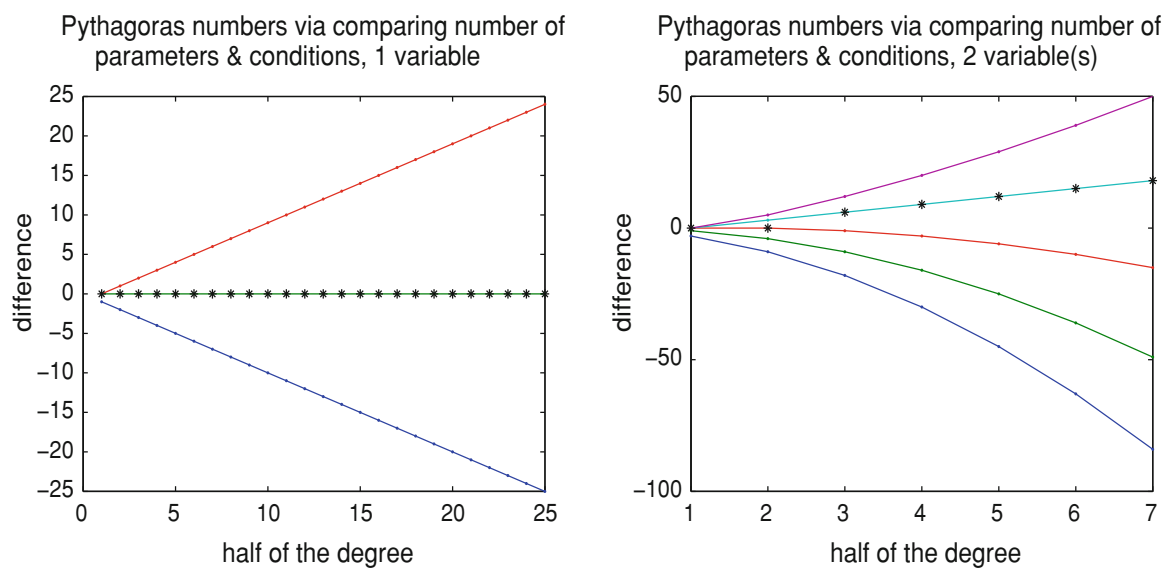

Fig. 1 Pythagoras number of sos-polynomials for $n=1$ (on the left) and $n=2$ (on the right) of total degree

$$
\begin{aligned}
& \pi_{T}(f)=\left\lceil\frac{2 \hat{e}+1-\sqrt{(2 \hat{e}+1)^{2}-8 \hat{a}}}{2}\right\rceil=\left\lceil\lambda_{T}(n+1,2 d)\right\rceil, \\
& \pi_{C}(f)=\left\lceil\frac{2(d+1)^{n}+1-\sqrt{\left[2(d+1)^{n}+1\right]^{2}-8(2 d+1)^{n}}}{2}\right\rceil=\left\lceil\lambda_{C}(n+1,2 d)\right\rceil .
\end{aligned}
$$

We will see in the next section an algorithm for finding the Pythagoras numbers. Let us now give some examples for the total degree case. The different lines in Fig. 1 show the difference $\mu(\hat{e}, r)-\hat{a}$ in function of the half of the degree for subsequent values of $r=1,2, \ldots$ The "black-star" point corresponding to each value of degrees gives us the Pythagoras number for such degree value. The univariate polynomial case is represented on the left figure. For each value of half of is represented on the left figure the degree $d$, there are many values of difference $\mu(\hat{e}, r)-\hat{a}$ satisfying inequality (10). The "black-star" point is the first one satisfying (10) and it is on the second line, counted up, of those points. This gives us the value of Pythagoras number $r=2$. This gives us the well-known result that $\pi_{T}(1,2 d)=2, \forall d \in \mathbb{N}$. Similarly, the bivariate case is on the right figure, the Pythagoras numbers corresponding to $d=1,2,3, \ldots, 7$ respectively, are $r=3,3,4, \ldots, 4$.

Similarly fix each $n=1, \ldots, 5$, the quantities of the conjecture on the Pythagoras number in function of the half of the degree are shown in the left figure of Fig. 2. The points on the lowest line (blue line) represent the Pythagoras number of univariate polynomial corresponding to the half of the degrees $d=1, \ldots, 30$. It turns out that $\pi_{T}(1,2 d)=2, \forall d=1, \ldots, 30$ and this is well-known in literature. For the other case, we see that $\pi_{T}(2,60)=4, \pi_{T}(3,60)=8, \pi_{T}(4,60)=14, \pi_{T}(5,60)=26$. All these values of $r$ are determined as in (12). 

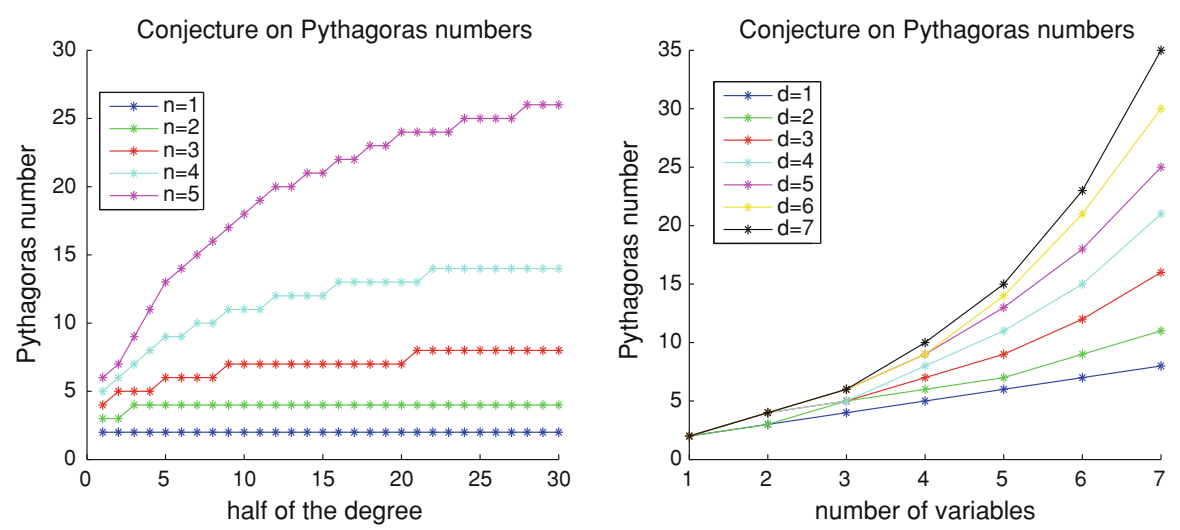

Fig. 2 Conjecture on Pythagoras number of sos-polynomials in function of the half of the total degree and in function of number of variables

A description of the relation between the number of variables and the difference $\mu(e, r)-a$ corresponding to the half of degree $d=1, \ldots, 7$ is showed in the right one of Fig. 2.

In the next section, we derive an algorithm that computes the Pythagoras number of a given (multivariate) sos-polynomial. Applying this algorithm on several random sos-polynomials numerically confirms the conjecture as shown in Sect. 5.

\section{Algorithm for finding the Pythagoras number}

To proceed our algorithm, the equations in (9) need to be reformulated as follows. Let $M_{n}^{2 d}$ be the $(\hat{a} \times n)$-matrix, where the $k$-th row is the vector $\gamma \in \Gamma^{\Re}(n, d)$ and let $v$ be the column vector with the $l$-th coordinate $(2 d+1)^{l-1}, l=0,1, \ldots, n-1$. Then it is not difficult to see the coordinates of $M_{n}^{2 d} v$ are pairwise distinct. Moreover, for each $\gamma \in \Gamma^{\Re}(n, d)$, let $L_{\gamma}=\{(\alpha, \beta): \alpha+\beta=\gamma, \alpha, \beta \in \Omega(n, d)\}$, then

$$
L_{\gamma}=\left\{(\alpha, \beta):\left(M_{n}^{2 d} v\right)_{k}=(\alpha+\beta) v\right\}
$$

and the equations in (9) are rewritten as follows

$$
f_{\gamma}=\sum_{(\alpha, \beta) \in L_{\gamma}}\left(\sum_{l=1}^{r} h_{l \alpha} h_{l \beta}\right), \quad k=1, \ldots, \bar{\omega} .
$$

The main idea of the algorithm is as follows. For each pair of natural numbers $(n, d)$ which are greater than or equal to 1 , we randomly choose a sos-polynomial $f(x) \in \mathbb{R}[x]_{2 d}$ as in (8).

As discussed at the end of Sect. 2, without loss generality, $f(x)$ can be chosen as a sum of $\omega$ squares. One way to determine such a sos-polynomials $f$ is $f(x)=$ $\mathbf{x}_{d}^{T} A \mathbf{x}_{d}$, for some $(\omega \times \omega)$-matrix $A$. So, the coefficients of $f$ can be computed as 
$f(x)=\mathbf{x}_{d}^{T} A \mathbf{x}_{d}=\sum_{\gamma \in \Gamma^{\Re}(n, d)} f_{\gamma} x^{\gamma}$. We then solve the system (13) step-by-step for $r=1,2, \ldots, \omega$. The smallest number $r$ such that (13) has a solution is the Pythagoras number we want to find.

Our algorithm is implemented in Mатцав. The system of nonlinear equations (13) is solved by using the Levenberg-Marquardt method [11] implemented by "fsolve". In this sense, the system of nonlinear equations (13) is equivalent to the problem of finding an $(\omega \times r)$-matrix $H$ with $\operatorname{rank}(H)=r$ and $\left(H H^{T}\right)_{\alpha \beta}=\sum_{l=1}^{r} h_{l \alpha} h_{l \beta}$ satisfying (13). In the algorithm, we need an upper bound of the residual as follows.

Let $F: \mathbb{R}^{\omega \times r} \rightarrow \mathbb{R}^{\bar{\omega}}$ be the function with coordinate functions are determined by

$$
F_{\gamma}(H)=\left(\sum_{(\alpha, \beta) \in L_{\gamma}} \sum_{l=1}^{r} h_{l \alpha} h_{l \beta}\right)-f_{\gamma}, \forall \gamma \in \Gamma^{\Re}(n, d) .
$$

The matrix $H^{(r)}, r=1, \ldots, \omega$, solving (13) is found numerically if $\left\|F\left(H^{(r)}\right)\right\|_{2}<\tau$ for some small enough positive real number $\tau$. The Levenberg-Marquardt algorithm, corresponding to $r$, stops at iteration $k \geq 1$ if the solution $H_{[k]}^{(r)}$ is numerically determined such that

$$
\frac{\left\|\left|F\left(H_{[k]}^{(r)}\right)\left\|_{2}^{2}-\right\| F\left(H_{[k-1]}^{(r)}\right) \|_{2}^{2}\right|\right.}{\left\|F\left(H_{[k-1]}^{(r)}\right)\right\|_{2}^{2}} \leq \hat{\tau}
$$

for some $\hat{\tau}>0$. Note that the objective function is nonconvex and hence a nonlinear least squares solver may indeed get stuck in local minima. However, when we find a solution for which the residuals vanish for a certain choice of the rank, we know that the Pythagoras number is bounded above by that rank. Moreover, we often find that this bound coincides with our conjecture on the Pythagoras number.

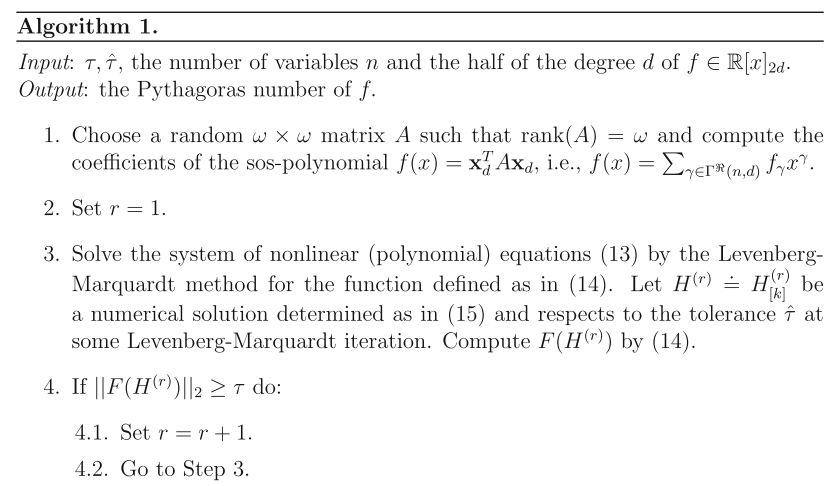

The algorithm stops at $r \leq e$ such that $\left\|F\left(H^{(r)}\right)\right\|_{2}<\tau$ is satisfied for the first time and the Pythagoras number is $\pi(f)=r$ and a numerical representation of $f$ is $f(x)=\mathbf{x}_{d}^{T}\left(H^{(r)} H^{(r)^{T}}\right) \mathbf{x}_{d}$.

In our experiment, the lexicographic order " $\leq$ " over the ring $\mathbb{R}\left[x_{1}, \ldots, x_{n}\right]$ is applied. Recall that, for any two monomials $x^{\alpha}=x_{1}^{\alpha_{1}}, \ldots, x_{n}^{\alpha_{n}}, x^{\beta}=x_{1}^{\beta_{1}}, \ldots, x_{n}^{\beta_{n}}$ 
where $\alpha, \beta \in \mathbb{N}^{n}$, this order is defined by

$$
\begin{aligned}
x^{\alpha} \leq x^{\beta} & \Longleftrightarrow \alpha \leq \beta \\
& \Longleftrightarrow\left[\begin{array}{l}
\alpha=\beta, \\
\exists k=1, \ldots, n: \alpha_{k}<\beta_{k} \text { and } \alpha_{i}=\beta_{i}, \forall i<k
\end{array}\right.
\end{aligned}
$$

The experiment shows that, when the threshold $\tau$ is made smaller, the computed number $r$ might become bigger and bigger. For example, for the total degree, the numerically determined Pythagoras number might be greater than $\hat{e}$. This is because the optimization method Levenberg-Marquardt is executed in finite precision. In this case, the Pythagoras number is determined to be $r$ such that $H^{(r)}$ satisfies (13) and the absolute value of the gap of the residuals $\left\|F\left(H^{(s)}\right)\right\|_{2}$ at $s=r$ and $s=r-1$ is the maximal value of all the gaps determined at each pair $(s, s+1)$ for all $s \geq r$.

\section{Numerical verification}

In Table 1, we show the Pythagoras numbers $\pi_{T}(n, 2 d)$ corresponding to the number of variables $n$ and the total degree of the polynomials $2 d$. For all cases of $(n, d)$, we fix $\tau=10^{-9}, \hat{\tau}=10^{-6}$. Additionally, we also show their corresponding lower and upper bounds $\lambda_{T}(n, 2 d), \Lambda_{T}(n, 2 d)$ of (5) for a comparison. As mentioned in Sect. 2, we note that $\pi_{T}(n, 2 d)=p_{T}(n+1,2 d)$. The numerical result for the coordinate degree case is showed in Table 2. Tables 1 and 2 show that $\pi_{C}(n, 2 d) \leq \pi_{T}(n, 2 n d)$. For example, $\pi_{C}(4,4)=9 \leq 10=\pi_{T}(4,16)$. From these tables, it turns out that the Pythagoras numbers are much smaller than their corresponding upper bounds. These tables also show the corresponding number of singular values numerically equal to zero of the Jacobian matrix in the Levenberg-Marquardt iteration at which Algorithm 1 stops. Because these numbers do not influence the Pythagoras number determined numerically, we postpone the discussion of these numbers to Sect. 6.3. We would like to emphasize that all Pythagoras numbers conjectured in Sect. 3 are confirmed by the numerical experiments with the results shown in Tables 1 and 2.

\section{Pythagoras number of (complex) multivariate Laurent polynomials positive on the $n$-torus}

In this section, we derive a conjecture on the exact value of the Pythagoras number of multivariate Laurent polynomials that can be represented as a sum of square magnitudes of complex-coefficient polynomials on the $n$-torus $\mathbb{T}^{n}$. Similar to the case of sos-polynomials, the Pythagoras number is the minimal rank of a low-rank representation of such a positive Laurent polynomial. We present a numerical optimization method to determine such a minimal low-rank representation. As before, we will consider the Pythagoras number for the total degree as well as the coordinate degree.

We first summarize some results on Laurent polynomial. Given $1 \leq n, d \in \mathbb{N}$. Similar to (4), let 
Table 1 Numerical values for the Pythogaras numbers $\pi_{T}(n, 2 d)$ for some values of $n$ and $d$ with the corresponding lower bounds $\lambda_{T}(n+1,2 d)$ and upper bounds $\Lambda_{T}(n+1,2 d)$ and corresponding zero singular values of Jacobian matrix in the Levenberg-Marquardt step

\begin{tabular}{|c|c|c|c|c|c|}
\hline$n$ & $d$ & $\pi_{T}(n, 2 d)$ & $\lambda_{T}(n+1,2 d)$ & $\Lambda_{T}(n+1,2 d)$ & $\sigma$ \\
\hline 1 & $1,2,3, \ldots$ & 2 & 2 & $2,2.702,3.275, \ldots$ & 1 \\
\hline \multirow[t]{5}{*}{2} & 1 & 3 & 3 & 3 & 3 \\
\hline & 2 & 3 & 3 & 5 & 3 \\
\hline & 3 & 4 & 3.135 & 7 & 12 \\
\hline & 4 & 4 & 3.242 & 9 & 15 \\
\hline & $5, \ldots, 11$ & 4 & $3.327, \ldots, 3.598$ & $11, \ldots, 23$ & $18, \ldots, 36$ \\
\hline \multirow[t]{6}{*}{3} & 1 & 4 & 4 & 4 & 6 \\
\hline & 2 & 5 & 4.156 & 7.882 & 15 \\
\hline & 3 & 5 & 4.618 & 12.471 & 16 \\
\hline & 4 & 5 & 5 & 17.673 & 10 \\
\hline & $5,6,7,8$ & 6 & $5.312, \ldots, 5.962$ & $23.422, \ldots, 43.526$ & $50, \ldots, 21$ \\
\hline & $9, \ldots, 14$ & 7 & $6.117, \ldots, 6.638$ & $51.078, \ldots, 94.317$ & $210, \ldots, 265$ \\
\hline \multirow[t]{8}{*}{4} & 1 & 5 & 5 & 5 & 10 \\
\hline & 2 & 6 & 5.488 & 11.343 & 20 \\
\hline & 3 & 7 & 6.513 & 20 & 35 \\
\hline & 4 & 8 & 7.411 & 30.968 & 65 \\
\hline & 5 & 9 & 8.177 & 44.247 & 133 \\
\hline & 6 & 9 & 8.831 & 59.835 & 70 \\
\hline & 7 & 10 & 9.392 & 77.732 & 240 \\
\hline & 8 & 10 & 9.876 & 97.939 & 105 \\
\hline \multirow[t]{5}{*}{5} & 1 & 6 & 6 & 6 & 15 \\
\hline & 2 & 7 & 7.000 & 15.382 & 21 \\
\hline & 3 & 9 & 8.874 & 29.902 & 42 \\
\hline & 4 & 11 & 10.620 & 50.237 & 99 \\
\hline & 5 & 13 & 12.187 & 77.000 & 273 \\
\hline \multirow[t]{4}{*}{6} & 1 & 7 & 7 & 7 & 21 \\
\hline & 2 & 9 & 8.695 & 20.000 & 42 \\
\hline & 3 & 12 & 11.752 & 42.491 & 84 \\
\hline & 4 & 15 & 14.785 & 77.000 & 147 \\
\hline
\end{tabular}

$$
\Gamma^{\Im}(n, d)=\Omega(n, d)-\Omega(n, d)=\{\alpha-\beta: \alpha, \beta \in \Omega(n, d)\} .
$$

$\Gamma^{\Im}(n, d)$ be the set of all $n$-tuples $\gamma \in \mathbb{N}^{n}$ which are the possible exponents of the Laurent polynomials. We suppose $\Gamma^{\Im}(n, d)$ is endowed with an ordering " $\leq$ " and its elements are indexed in the ascending way. That is, if the elements are indexed as $\Gamma^{\Im}(n, d)=\left\{\gamma^{(1)}, \gamma^{(2)}, \ldots\right\}$ then $\gamma^{(i)} \leq \gamma^{(i+1)}, \forall i$ under that ordering. It is certain that $\Omega(n, d) \subset \Gamma^{\Im}(n, d)$ and we assume that $\Omega(n, d)$ is also equipped with the ordering restricted by the one on $\Gamma^{\Im}(n, d)$ and its elements are also indexed in ascending order. 
Table 2 Numerical values for the Pythogaras numbers $\pi_{C}(n, 2 d)$ for some values of $n$ and $d$ with the corresponding lower bounds $\lambda_{C}(n+1,2 d)$ and corresponding zero singular values of Jacobian matrix in the Levenberg-Marquardt step

\begin{tabular}{lllll}
\hline$n$ & $d$ & $\pi_{C}(n, 2 d)$ & $\lambda_{C}(n+1,2 d)$ & $\sigma$ \\
\hline 1 & $1,2,3, \ldots$ & 2 & 2 & 1 \\
2 & 1 & 3 & 3 & 3 \\
& 2 & 4 & 3.156 & 11 \\
& 3 & 4 & 3.300 & 15 \\
& 4 & 4 & 3.404 & 19 \\
& $5, \ldots, 11$ & 4 & $3.481, \ldots, 3.709$ & $23, \ldots, 47$ \\
3 & 1 & 5 & 4.228 & 13 \\
& 2 & 5 & 5.000 & 10 \\
& 3 & 6 & 5.557 & 41 \\
& 4 & 6 & 5.950 & 21 \\
4 & 1 & 6 & 6 & 15 \\
& 2 & 9 & 8.068 & 104 \\
& 3 & 10 & 9.538 & 21 \\
5 & 1 & 9 & 8.620 & 45 \\
6 & 2 & 14 & 13.190 & 277 \\
& 1 & 13 & 12.517 & 103 \\
\hline
\end{tabular}

We will consider Laurent polynomials in $n$ variables of the form

$$
p(z)=p\left(z_{1}, \ldots, z_{n}\right)=\sum_{\gamma \in \Gamma^{\Im}(n, d)} p_{\gamma} z^{\gamma}
$$

where $z=\left(z_{1}, \ldots, z_{n}\right) \in \mathbb{C}^{n}, \gamma=\left(\gamma_{1}, \ldots, \gamma_{n}\right) \in \mathbb{N}^{n}, z^{\gamma}=z_{1}^{\gamma_{1}} \ldots z_{n}^{\gamma_{n}}$ and $p_{\gamma} \in \mathbb{C}$. We suppose the coefficients of $p(z)$ are written corresponding to the arrangement of exponents in $\Gamma^{\Im}(n, d)$. Our numerical experiment will deal with the two following types of Laurent polynomials. The first type consists of Laurent polynomials in $n$ variables of total degree $d$ corresponding to $\Omega_{T}(n, d)$. The set of all exponents of the polynomial as in (18) is hence obtained as

$$
\Gamma_{T}^{\Im}(n, d)=\left\{\gamma \in \mathbb{Z}^{n}: 0 \leq|\gamma|=\sum_{n=1}^{n}\left|\gamma_{i}\right| \leq d,\left|\gamma_{i}\right| \leq d, \forall i=1, \ldots, n\right\}
$$

The second type is of Laurent polynomials in $n$ variables of coordinate degree $d$ as described in $[7,10]$ and defined as follows

$$
p(z)=\sum_{k_{1}=-d}^{d} \ldots \sum_{k_{n}=-d}^{d} p_{k_{1} \cdots k_{n}} z_{1}^{k_{1}} \ldots z_{n}^{k_{n}}=\sum_{\gamma \in \Gamma_{C}^{\Im}(n, d)} p_{\gamma} z^{\gamma}
$$


where

$$
\Gamma_{C}^{\Im}(n, d)=\left\{\gamma \in \mathbb{Z}^{n}:\left|\gamma_{i}\right| \leq d, \forall i=1, \ldots, n\right\}
$$

It is clear that the univariate cases of the two above kinds coincide. Moreover, if an univariate Laurent polynomial $p(z)$ of degree $d$ is nonnegative on the unit circle $\mathbb{T}$ then it is sosm due to the Fejér-Riesz Theorem. Namely, there is a polynomial $q(z)=\sum_{l=0}^{d} q_{l} z^{l}$ with complex coefficients such that $p(z)=|q(z)|^{2}, \forall z \in \mathbb{T}$. This yields that any sum of square magnitudes of univariate polynomials can be represented as only one square magnitude of a polynomial. For the case of the multivariate polynomials, Dritschel [6] proved that any (strictly) positive Laurent polynomial on the $n$-torus $\mathbb{T}^{n}$ can be represented as a sum of square magnitudes of polynomials. Calderon and Perpinsky [3] and Rudin [17] proved that it is not always true for the case the polynomial is nonnegative on the torus $\mathbb{T}^{n}, n \geq 2$. Additionally, Mclean and Woerdeman [10] proposed an algorithm to check whether a given Laurent polynomial can be represented as a sum of square magnitudes of polynomials and if it is the case, find square magnitude terms via spectral factorization. Geronimo and Ming-Jun Lai [7] proposed another algorithm based on the reducing problem of factorization of multivariate polynomials with coordinate degree to the univariate problem.

However, the algorithms above do not show how a sosm-polynomial can be represented as a sum of square magnitudes of polynomials with the number of terms as small as possible. That is, given an sosm-polynomial $p(z)$ of the form

$$
p(z)=\sum_{k=1}^{m}\left|p_{k}(z)\right|^{2}, \quad \forall z \in \mathbb{T}^{n},
$$

where

$$
p_{k}(z)=\sum_{\alpha \in \Omega(n, d)} p_{k \alpha} z^{\alpha} \in \mathbb{C}[z], \quad \forall k=1, \ldots, m,
$$

can $p(z)$ be represented as a sum of $r \leq m$ square magnitudes? We propose to call the smallest number determined in such way Pythagoras number of the sosm-(Laurent) polynomial. The Pythagoras number was defined for the commutative ring with unit in the literature. In this article, we define the Pythagoras number of sosm-polynomials on the $n$-torus as follows.

Definition 2 Let $f$ be a sosm-(Laurent) polynomial in $n$ variables and of degree $d$ defined on the $n$-torus $\mathbb{T}^{n}$. The Pythagoras number of $f$, say $\pi^{\Im}(f)$, is the smallest positive integer number such that $f$ can be represented as a sum of $\pi^{\Im}(f)$ square magnitudes of polynomials. Pythagoras number of a subset $L$ of the set of all Laurent polynomials defined on the $n$-torus $\mathbb{T}^{n}$ is defined to be the supremum of the Pythagoras numbers of all sosm-Laurent polynomials in $L$ and is writen $\pi^{\Im}(L)$.

Analogously to sos-polynomials, we derive the following proposition which gives us the connection between the sosm-polynomials of total degree and the ones of coordinate degree. 
Proposition 3 (i) Any sosm-polynomial $f$ in $n$ variables and of coordinate degree $d$ is always a sosm-polynomial of total degree $n d$;

(ii) $\pi_{C}^{\Im}(f)=\pi_{T}^{\Im}(f)$;

(iii) $\pi_{C}^{\Im}(n, d) \leq \pi_{T}^{\Im}(n, n d)$, where $\pi_{C}^{\Im}(n, d),\left(\pi_{T}^{\Im}(n, d)\right)$ denotes Pythagoras number of the set of all sosm-polynomials in $n$ variables and of coord (respectively, total) degree.

In Sect. 6.2, we present a method of computing numerically the coefficients of such square magnitude terms in a minimal low-rank representation. The way to obtain a system of nonlinear equations is similar to the case of real polynomials in Sect. 4. However, the command "fsolve" in Matlab can only be used for system of real variables and not for a complex system. Our method in this case then is based on the generalized Levenberg-Marquardt method [18] for solving the least squares problem in complex variables.

\subsection{Matrix representation of sosm-polynomials}

We return to the sets $\Gamma^{\Im}(n, d)$ and $\Omega(n, d)$ with a given ordering " $\leq$ ". By $\mathbf{z}$ denotes the vector of all possible monomials with exponents in $\Omega(n, d)$. Now, suppose

$$
q(z)=\sum_{\alpha \in \Omega(n, d)} q_{\alpha} z^{\alpha} \in \mathbb{C}[z] .
$$

Then

$$
|q(z)|^{2}=\overline{q(z)} q(z)=\mathbf{z}^{H}\left(\overline{\mathbf{q}} \mathbf{q}^{T}\right) \mathbf{z}=\mathbf{z}^{H} Q \mathbf{z},
$$

where $\mathbf{z}^{H}=\overline{\mathbf{z}}^{T}, Q$ is a semidefinite Hermitian matrix of order $|\Omega(n, d)|$, the over-line denotes the element-wise conjugate operator of complex vectors, ${ }^{T}$ denotes the transposition of vectors and $\mathbf{q}$ denotes the column vector of all coefficients of polynomial $q(z)$.

Now, suppose a Laurent polynomial $p(z)$ is given of the form (20), we want to find a minimal number $r$ and corresponding complex polynomials $q_{1}, \ldots, q_{r} \in \mathbb{C}[z]$ of the form (21) such that

$$
p(z)=\sum_{k=1}^{r}\left|q_{k}(z)\right|^{2}, \quad \forall z \in \mathbb{T}^{n} .
$$

We have that

$$
p(z)=\sum_{k=1}^{r}\left|q_{k}(z)\right|^{2}=\mathbf{z}^{H}\left(\sum_{k=1}^{r} \overline{\mathbf{q}}_{k} \mathbf{q}_{k}^{T}\right) \mathbf{z}=\mathbf{z}^{H}\left(\bar{G} G^{T}\right) \mathbf{z},
$$

where $G=\left[\mathbf{q}_{1}, \ldots, \mathbf{q}_{r}\right]$ is a $(|\Omega(n, d)| \times r)$-matrix and $\bar{G}$ is the element-wise conjugate of $G$. Hence, the relation between the coefficients of the sosm-Laurent 
polynomials $p(z)$ and the polynomials $q_{k}$ can be obtained as follows

$$
\sum_{\substack{\beta-\alpha=\gamma \\ \alpha, \beta \in \Omega(n, d)}}\left(\sum_{k=1}^{r} \bar{q}_{k \alpha} q_{k \beta}\right)=p_{\gamma}, \forall \gamma \in \Gamma^{\Im}(n, d),
$$

where $q_{k \alpha}$ denotes the $\alpha$-th coefficient of the polynomial $q_{k}(z)$.

We will see in the next two subsections that the solution of the system (24) can be found as a minimizer of a nonlinear least squares problem. To solve this least squares problem, we use an implementation of the generalized Levenberg-Marquardt method [18].

6.2 Solving a system of complex nonlinear equations via the generalized

Levenberg-Marquardt method

Because $p(z)$ is a sum of square magnitudes, $p_{-\gamma}=\bar{p}_{\gamma}, \forall \gamma \in \Gamma^{\widetilde{\Im}}(n, d)$ and the number of equations of the system (24) is hence reduced as $\left\lfloor\left|\Gamma^{\Im}(n, d)\right| / 2\right\rfloor+1$, where $\lfloor k\rfloor$ denotes the largest integer not greater than $k$. That is, it is sufficient to consider the system

$$
\sum_{\substack{\beta-\alpha=\gamma \\ \alpha, \beta \in \Omega(n, d)}}\left(\sum_{k=1}^{r} \bar{q}_{k \alpha} q_{k \beta}\right)=p_{\gamma}, \quad \forall \gamma \in \Gamma^{\Im}(n, d), \gamma \geq 0 .
$$

Here, " $\gamma \geq 0$ " means $\gamma$ is greater than the zero element in $\Gamma^{\Im}(n, d)$ corresponding to the given ordering " $\leq$ ".

We first give a similar conjecture on the Pythagoras number of sosm-polynomials. Again, the Pythagoras number in this conjecture is determined as the smallest number $r$ such that the number of unknowns in the system (25) is not less than the number of equations. To give this conjecture, one can view the complex system (25) as a system of real parameters as follows. Suppose the matrix $\bar{G}$ as in (23) is full rank, applying LQ factorization for this matrix, we have $\bar{G}=\bar{L} Q$. Here, $Q$ is a unitary matrix and the complex conjugate of $L$ is of the form

$$
\bar{L}=\left(\begin{array}{ccc}
* & & \\
\vdots & \ddots & \\
* & \ldots & * \\
\vdots & \ddots & \vdots \\
* & \ldots & *
\end{array}\right)
$$

where $*$ denotes possible nonzero entries. We can then suppose $\bar{G}$ is of the form $\bar{L}$ and note that $\bar{L}$ can be taken such that each diagonal element on any of its columns is real. So, if each complex variable of the system (25) is viewed as a pair of two real 
ones then $\bar{L}$ contains

$$
2\left(|\Omega(n, d)| r-\frac{r(r-1)}{2}\right)-r=2|\Omega(n, d)| r-r^{2}
$$

real variables. For the number of equations of (25), we note that $p_{0}, 0 \in \Gamma^{\Im}(n, d)$ is real and the cardinality of $\Gamma^{\mathfrak{S}}(n, d)$ is always an odd number. Hence, the system (25) consists of

$$
2\left(\left\lfloor\frac{\left|\Gamma^{\Im}(n, d)\right|}{2}\right\rfloor+1\right)-1=\left|\Gamma^{\Im}(n, d)\right|
$$

equations. We thus have the following conjecture.

Conjecture 2 The Pythagoras number of the sosm-polynomial (20) is the smallest number $r$ such that

$$
\left|\Gamma^{\Im}(n, d)\right| \leq 2|\Omega(n, d)| r-r^{2} .
$$

Equivalently,

$$
r=\left\lceil|\Omega(n, d)|-\sqrt{|\Omega(n, d)|^{2}-\left|\Gamma^{\Im}(n, d)\right|}\right\rceil
$$

For the coordinate degree case, the above condition is translated into

$$
r=\left\lceil(d+1)^{n}-\sqrt{(d+1)^{2 n}-(2 d+1)^{n}}\right\rceil .
$$

For the case of total degree, we have $\left|\Omega_{T}(n, d)\right|=\left(\begin{array}{c}n+d \\ d\end{array}\right)=e(n+1,2 d)$. The number of elements of $\Gamma_{T}^{\widetilde{\Im}}(n, d)$ is determined numerically. Figure 3 shows the conjectures on the Pythagoras number of sosm-polynomials of total degree. The figure on the left conjectures the Pythagoras number in function of degree corresponding to each number of variables $n=1,2,3$. The other one shows the conjectures on the Pythagoras number in function of number of variables. The conjecture above is now confirmed by the numerically solving the system (25) as follows.

To solve the system (25), we apply the generalized Levenberg-Marquardt method [18] to solve the nonlinear least squares problem $\min _{\mathbf{q}} \frac{1}{2}\left\|F\left(\begin{array}{c}\mathbf{q} \\ \mathcal{C}\end{array}\right)\right\|^{2}$, where the function $F: \mathcal{C} \rightarrow \mathbb{C}^{\left\lfloor\Gamma^{\Im}(n, d) \mid / 2\right\rfloor+1}$ is defined by the component functions

$$
\left.F_{y} \stackrel{\mathcal{C}}{q}\right)=\sum_{\substack{\beta-\alpha=\gamma \\ \alpha, \beta \in \Omega(n, G)}}\left(\sum_{k=1}^{r} \bar{q} k \alpha q k \beta\right)-p \gamma, \quad \forall \gamma \in \Gamma(n, d), \gamma \geq 0,
$$

$c=\left\{\stackrel{\mathcal{C}}{q}=(q, \bar{q}): q \in \mathbb{C}^{v}\right\} \subset \mathbb{C}^{2 v}, v=|\Omega(n, d)| r$ and $\mathbf{q}=\left[\mathbf{q}_{1}^{T}, \ldots, \mathbf{q}_{r}^{T}\right]^{T}$ is a column vector of the coefficients of all $r$ polynomials $q_{1}(z), \ldots, q_{r}(z)$. 


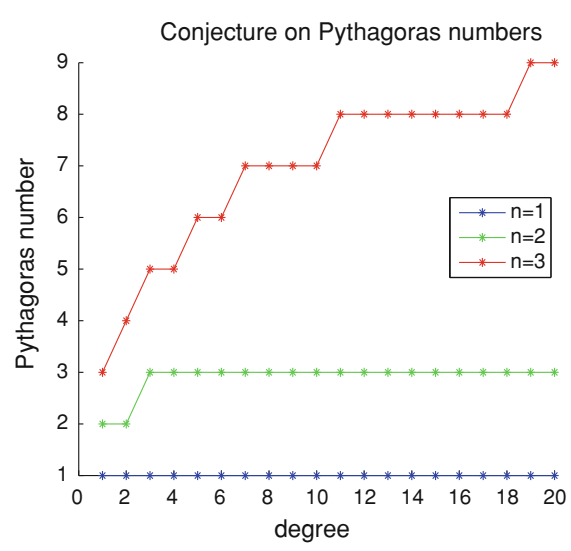

Conjecture on Pythagoras numbers

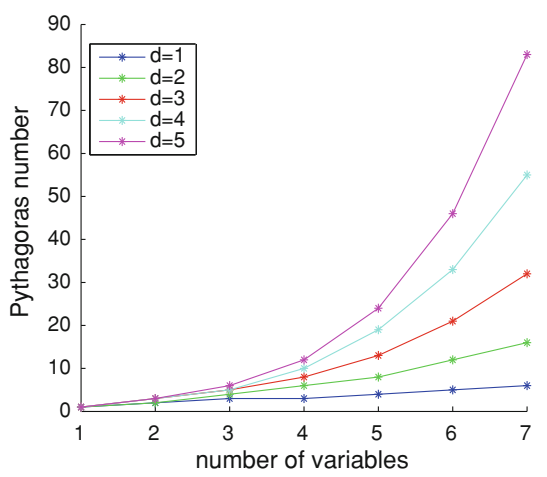

Fig. 3 Conjecture on the Pythagoras number of sosm-polynomials in function of degree (on the left) and in function of number of variables (on the right)

The algorithm solves the system of $\left\lfloor\frac{\left|\Gamma^{\Im}(n, d)\right|}{2}\right\rfloor+1$ equations and with $|\Omega(n, d)| r$ complex variables which are the coefficients of $r$ polynomials $q_{1}(z), \ldots, q_{r}(z)$. We note that $\left|\Omega_{T}(n, d)\right| \leq\left|\Omega_{C}(n, d)\right|$ and hence $\left|\Gamma_{T}^{\widetilde{\Im}}(n, d)\right| \leq\left|\Gamma_{C}^{\widetilde{\Im}}(n, d)\right|$. Similarly to the case of sos-polynomials, a tolerance for the $k$-iteration of the Levenberg-Marquardt algorithm $\hat{\tau}$ is given such that

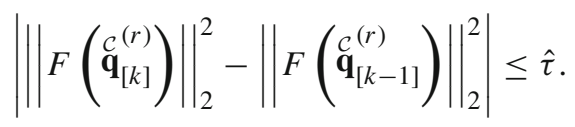

Additionally, a tolerance $\tau$ for the algorithm is also needed.

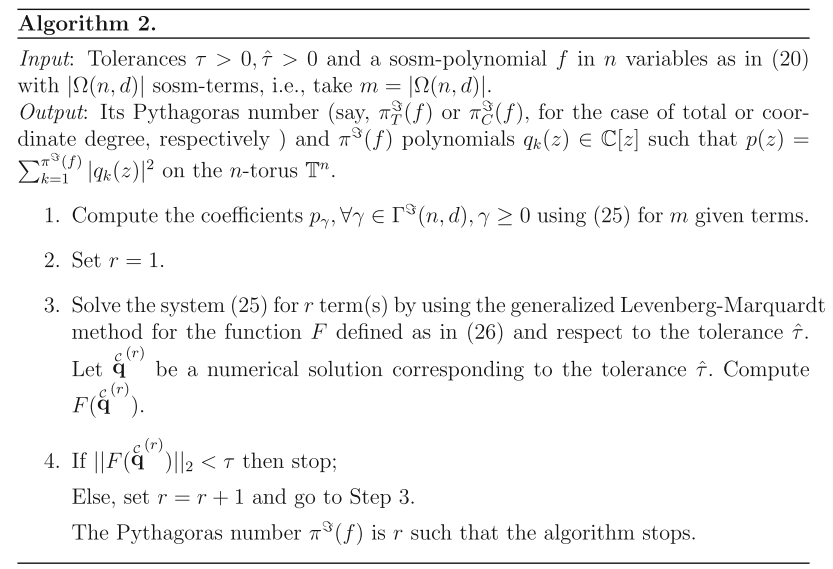

\subsection{Numerical results}

Our algorithm is implemented in Matlab. The tolerances are chosen as $\tau=\hat{\tau}=$ $10^{-6}$ for all cases in that table. Table 3 shows the results. The numerical experiment 
Table 3 Numerical values of the Pythagoras number of sosm-polynomials of total or coordinate degree and the corresponding lower bound $\lambda^{\Im}(n, d)=|\Omega(n, d)|-\sqrt{|\Omega(n, d)|^{2}-\left|\Gamma^{\Im}(n, d)\right|}$ as in Conjecture 2 and corresponding zero singular values of Jacobian matrix in the Levenberg-Marquardt step

\begin{tabular}{|c|c|c|c|c|}
\hline$n$ & $d$ & $\pi_{T}^{\Im}(n, d)$ & $\lambda_{T}^{\widetilde{\Im}}(n, d)$ & $\sigma$ \\
\hline 1 & $\forall d$ & 1 & 1 & 1 \\
\hline \multirow[t]{2}{*}{2} & 1,2 & 2 & $1.586,1.877$ & 5,5 \\
\hline & $3, \ldots, 20$ & 3 & $2.063, \ldots, 2.746$ & $23, \ldots, 125$ \\
\hline \multirow[t]{4}{*}{3} & 1 & 3 & 2.268 & 11 \\
\hline & 2 & 4 & 3.292 & 25 \\
\hline & 3,4 & 5 & $4.094,4.735$ & 53,41 \\
\hline & 5 & 6 & 5.256 & 11 \\
\hline 4 & 1 & 3 & 3 & 9 \\
\hline 5 & 1 & 4 & 3.764 & 17 \\
\hline 6 & 1 & 5 & 4.551 & 27 \\
\hline 7 & 1 & 6 & 5.354 & 39 \\
\hline $\mathrm{n}$ & $\mathrm{d}$ & $\pi_{C}^{\Im}(n, d)$ & $\lambda_{C}^{\widetilde{S}}(n, d)$ & $\sigma$ \\
\hline 1 & $\forall d$ & 1 & 1 & 1 \\
\hline \multirow[t]{2}{*}{2} & 1 & 2 & 1.354 & 7 \\
\hline & $2, \ldots, 12$ & 2 & $1.517, \ldots, 1.859$ & $11, \ldots, 51$ \\
\hline \multirow[t]{3}{*}{3} & 1 & 3 & 1.917 & 21 \\
\hline & $2, \ldots, 4$ & 3 & $2.424, \ldots, 2.951$ & $37, \ldots, 21$ \\
\hline & 5 & 4 & 3.103 & 397 \\
\hline \multirow[t]{2}{*}{4} & 1 & 3 & 2.771 & 15 \\
\hline & 2 & 5 & 3.995 & 185 \\
\hline 5 & 1 & 5 & 4.054 & 77 \\
\hline 6 & 1 & 7 & 5.974 & 167 \\
\hline 7 & 1 & 10 & 8.849 & 373 \\
\hline
\end{tabular}

shows that Conjecture 2 does not hold for the coordinate degree case. To investigate this further, we computed the number of singular values of the Jacobian that are numerically zero in the computed solution of the Levenberg-Marquardt algorithm. This number equals the number of free parameters that one loses for this sample of a sosm polynomial. For each $r$ in Algorithm 2, the number of free parameters must be greater than or equal to the number of equations. That is, Algorithm 2 stops at $r$ such that the following condition satifies

$$
2|\Omega(n, d)| r-\sigma \geq\left|\Gamma^{\Im}(n, d)\right|
$$

where $\sigma$ is the number of singular values of the Jacobian matrix that are numerically zero at the iteration at which the Levenberg-Marquardt step stops. The number $r$ determined by Algorithm 2 might be greater than the one in Conjecture 2. For example, in the right hand side table of Table 3 , for $(n, d)=(3,1)$, the number $r$ given by Conjecture 2 should be $r=2$ because the lower bound is 1.1917. But for $r=2$, the number 
of zero singular values of the Jacobian matrix in the last Levenberg-Marquardt step is 6 and, hence, (28) does not hold because $\left|\Omega_{C}(3,1)\right|=8,\left|\Gamma_{C}^{\Im}(3,1)\right|=27$. Similarly for the cases $(n, d)=(4,1),(4,2),(5,1),(6,1),(7,1)$. The approach shows that in the case of sosm-polynomials of total degree, the condition (28) for $r$ gives the same $r$ as Conjecture 2. Additionally, an analogous condition for sos-polynomials is defined as

$$
|\Omega(n, d)| r-\sigma \geq\left|\Gamma_{C}^{\Re}(n, d)\right|
$$

and this holds for all cases which are shown in Tables 1 and 2. The smallest $r$ satisfying this condition coincides with the $r$ given by Conjecture 1 .

\section{Conclusion}

We have proposed a method to numerically determine the Pythagoras number of either real multivariate sum of squares polynomials or multivariate sum of square magnitudes of polynomials on the $n$-torus $\mathbb{T}^{n}$. We have also given conjectures for the exact value of the Pythagoras number in these two cases. The values obtained by our numerical methods confirm these conjectures except for the sosm polynomials and the coordinate degree case. In this case, the number of free parameters that are lost, i.e., the number of singular values of the Jacobian that are numerically zero, is too large and a higher rank, giving the Pythagoras number, is needed. For sos-polynomials, the value of the Pythagoras number turns out to be much smaller than the upper bounds known in the literature. The value is the smallest integer just larger than the best lower bound in the literature. This could be an interesting observation for efficiently solving optimization problems over sets of nonnegative polynomials when such problems can be approximated well by optimization problems over sets of $\operatorname{sos}(\mathrm{m})$-polynomials.

\section{References}

1. Barvinok, A.I.: Problems of distance geometry and convex properties of quadratic maps. Discret. Comput. Geom. 13, 189-202 (1995)

2. Bochnack, J., Coste, M., Roy, M.-F.: Real Algebraic Geometry. Springer, Berlin (1998)

3. Calderon, A., Pataki, G.: On the phases of Fourier coefficients for positive real periodic functions. In: Perpinsky, R. (ed.) Computing Methods and Phase Problem in X-Ray Crystal, Analysis, pp. 339-346 (1952)

4. Choi, M.-D., Dai, Z.D., Lam, T.Y., Reznick, B.: Pythagoras number of some affine algebras and local algebras. Journal für die Reine und Angewandte Mathematik 1982(336), 45-82 (1982)

5. Choi, M.-D., Lam, T.Y., Reznick, B.: Sums of squares of real polynomials. In: Proceedings of Symposia in Pure Mathematics, vol. 58, pp. 103-126 (1995)

6. Dritschel, M.A.: On factorization of trigonometric polynomials. Integral Equ. Oper. Theory 49(1), 11-42 (2004) (in English)

7. Geronimo, J.S., Lai, M.-J.: Factorization of multivariate positive Laurent polynomials. J. Approx. Theory 139(1-2), 327-345 (2006)

8. Hilbert, D.: Über die Darstellung Definiter Formen als Summe von Formen-Quadraten. Math. Ann. 32, 342-350 (1888)

9. Lam, T.Y.: Algebraic Theory of Quadratic Forms, 2nd edn. Addison Wesley, Boston (1980)

10. Mclean, J.W., Woerdeman, H.J.: Spectral factorizations and sums of squares representation via semidefinite programming. SIAM J. Matrix Anal. Appl. 23, 646-655 (2001) (in English) 
11. Moré, J.J.: The Levenberg-Marquardt algorithm: implementation and theory. Lecture Notes Math. 630, 105-116 (1978)

12. Pataki, G.: On the rank of extreme matrices in semidefinite programs and the multiplicity of optimal eigenvalues. Math. Oper. Res. 23(2), 339-358 (May 1998)

13. Powers, V., Wörmann, T.: An algorithm for sums of squares of real polynomials. J. Pure Appl. Algebra 127, 99-104 (1998)

14. Prestel, A., Delzell, C.N.: Positive polynomials. Springer monographs in mathematics. Springer, Berlin (2001)

15. Rajwade, A.R.: Squares. Cambridge University Press, Cambridge (1993)

16. Robinson, R.M.: Some definite polynomials which are not sums of squares of real polynomials. In: Selected Questions of Algebra and Logic, pp. 264-282. Acad. Sci. USSR (1973)

17. Rudin, W.: The extension problem for positive-definite functions. Ill. J. Math. 7, 532-539 (1963)

18. Sorber, L., Van Barel, M., De Lathauwer, L.: Unconstrained optimization of real functions in complex variables. SIAM J. Optim. (accepted) 